\section{BRAZIULIAN JOURNAL \\ OF MEDICAL AND BIOLOGICAL RLSF.ARCH}

www.bjournal.com.br
ISSN 0100-879X

Volume 42 (10) 870-992 October 2009

BIOMEDICAL SCIENCES

AND

CLINICAL INVESTIGATION

Braz J Med Biol Res, October 2009, Volume 42(10) 968-972

\title{
Use of anti-PGL-1 antibodies to monitor therapy regimes in leprosy patients
}

E.M.R. Zenha, M.A.N. Ferreira and N.T. Foss

The Brazilian Journal of Medical and Biological Research is partially financed by

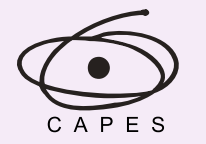

Ministério da Educação

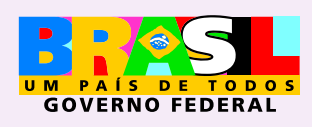

DFAPESP

Institutional Sponsors 


\title{
Use of anti-PGL-1 antibodies to monitor therapy regimes in leprosy patients
}

\author{
E.M.R. Zenha, M.A.N. Ferreira and N.T. Foss
}

Disciplina de Dermatologia, Departamento de Clínica Médica, Faculdade de Medicina de Ribeirão Preto, Universidade de São Paulo, Ribeirão Preto, SP, Brasil

\begin{abstract}
The suitability of IgM antibodies to PGL-1 for monitoring the response to multidrug therapy (MDT) was sequentially tested by ELISA in 105 leprosy patients, and bacterial indexes (BI) were also determined. Patients were divided into 3 groups: group 1, 34 multibacillary (MB) patients treated for 12 months with MDT-MB; group 2, 33 MB patients treated for 24 months with MDT$\mathrm{MB}$, and group 3, 38 paucibacillary (PB) patients treated for 6 months with MDT-PB. Untreated MB patients exhibited higher antibody levels (mean \pm SEM): group $1(6.95 \pm 1.35)$ and group $2(12.53 \pm 2.02)$ than untreated PB patients $(1.28 \pm 0.35)$. There was a significant difference $(P<0.01)$ in anti-PGL-1 levels in group 1 patients: untreated $(6.95 \pm 1.35)$ and treated for 12 months $(2.78 \pm 0.69)$ and in group 2 patients: untreated $(12.53 \pm 2.02)$ and treated for 24 months $(2.62 \pm 0.79)$. There was no significant difference between untreated $(1.28 \pm 0.35)$ and treated $(0.62 \pm 0.12)$ PB patients. Antibody levels correlated with BI. The correlation coefficient (Pearson's $r$ ) was 0.72 before and $0.23(P<0.05)$ after treatment in group 1 and 0.67 before and $0.96(P<0.05)$ after treatment in group 2 . BI was significantly reduced $(P<0.01)$ after 12 and 24 months on MDT (group 1: 1.26-0.26; group 2: 1.66-0.36). Our data indicate that monitoring anti-PGL-1 levels during MDT may be a sensitive tool for evaluating treatment efficacy. These data also indicate that the control of leprosy infection can be obtained with 12 months of MDT in MB patients.
\end{abstract}

Key words: Leprosy; Anti-PGL-1; Multidrug therapy; MDT 12 months; MDT 24 months

\section{Introduction}

Leprosy is a chronic infectious disease caused by Mycobacterium leprae characterized by a broad spectrum of clinical manifestations ranging from the relatively limited lesions of the tuberculoid form ( $T$ ) to the disseminative and progressive involvement of peripheral nerves, skin and other tissues of the lepromatous form (L). These polar forms are associated with the immunological response of the host to the infectious agent (1). Antibody production varies with the disease spectrum as a consequence of the bacillary load. Tuberculoid patients display lower levels of antibody production (2). In lepromatous patients, there is a lack of specific cell immune response to $M$. leprae and an increase in the humoral response, which does not play any protective role in leprosy (3).

Testing for the presence of the species-specific antibody to the M. leprae phenolic glycolipid-I (PGL-1) has been suggested as a method to detect new leprosy cases and for an early diagnosis of subclinical infection, since these antibodies reflect an early sign of antigenic stimulation (3-6). Although most studies of the incidence of leprosy in people seropositive for anti-PGL-1 have failed to demonstrate an increased risk, some investigators have reported an increase in the rate of detection of leprosy in seropositive contacts compared to seronegative contacts $(7,8)$.

ELISA has been used to detect anti-PGL-1 antibodies, mainly of the IgM class. Results from previous PGL-1 studies have demonstrated that the technique is sensitive for multibacillary leprosy but detects only 30 to $60 \%$ of paucibacillary cases (4). There is a significant correlation between IgM antibodies to PGL-1 and the bacterial index (BI), indicating that anti-PGL-1 IgM levels reflect the total bacillary load of leprosy patients and, thus, might be useful for monitoring patient response to antileprosy chemotherapy $(9,10)$. Serology could provide a method to monitor leprosy patients under treatment since the levels of anti-PGL-1 antibodies decrease during the administration of specific

Correspondence: N.T. Foss, Disciplina de Dermatologia, Departamento de Clínica Médica, FMRP, USP, 14049-900 Ribeirão Preto, SP, Brasil. E-mail: ntfoss@fmrp.usp.br

Research supported by FAEPA, CNPq-PIBIC, CNPq (\#300187/87-4, \#401001/2005-9) and Fundação Paulista Contra Hanseníase. Received February 14, 2009. Accepted August 11, 2009. Available online September 28, 2009. 
therapy (1). A serologic test for the diagnosis of subclinical infection and therapeutic follow-up has been applied, despite the high specificity and controversies surrounding the correlation of high levels of anti-PGL-1 antibodies with active, previous or future disease $(11,12)$.

Large numbers of new cases of leprosy have been detected in Brazil over the last years. Even though the coefficient of prevalence has decreased, about 30,590 new untreated cases were detected in 2007 (13). These data suggest that the Public Health Services should find new methods or therapeutic regimens for the control of the disease. Beside this increased detection of new cases of leprosy, it should be pointed out that about $60-70 \%$ of these cases (18,354-21,413 patients) are lepromatous, thus maintaining transmission of the disease (13).

The Health Ministry has defined new therapeutic regimens for leprosy in Brazil. Multibacillary (MB) patients should take the multidrug therapy (MDT-MB) with rifampicin (600 mg/month), clofazimine $(300 \mathrm{mg} / \mathrm{month}$ and $50 \mathrm{mg} /$ day) and dapsone (100 mg/day) for 12 months instead of 24 months (14). However, this short-term regimen was considered to be unsafe by some physicians and professionals working with the leprosy control program, even though a preliminary study showed no difference in leprosy treatment outcomes between 12- and 24-month multidrug regimens (15). No treatment changes have been tried for paucibacillary $(\mathrm{PB})$ patients, who continue to receive MDTPB with rifampicin (600 mg/month) and dapsone $(100 \mathrm{mg} /$ day) for 6 months (14).

In the present study, serum anti-PGL-1 IgM levels and the BI were measured sequentially in leprosy patients before and after treatment to evaluate the suitability of using the levels of IgM antibodies to PGL-1 to monitor the response to the classic (24 month) and short-term (12 month) MDT regimen.

\section{Patients and Methods}

\section{Patients}

One hundred and five leprosy patients were followed in the Division of Dermatology and National Leprosy Reference Center, University Hospital, Faculty of Medicine of Ribeirão Preto, University of São Paulo, Brazil. They were classified according to the spectrum of the disease as MB, all of whom with a positive $\mathrm{BI}$, and $\mathrm{PB}$, all of whom with a negative $\mathrm{BI}$.

The patients were divided into 3 groups: group 1, MB patients treated for 12 months with MDT (MDT-MB, shortterm regimen); group 2, MB patients treated for 24 months with MDT (MDT-MB, classic regimen), and group 3, PB patients treated with MDT-PB for 6 months. MB patients were treated with standard multidrug therapy, which included dapsone, rifampicin and clofazimine for 12 months or 24 months. PB patients also received the standard multidrug therapy with dapsone and rifampicin for 6 months.
Antibody determinations were performed on patients before and after MDT. Serum was collected by venipuncture, centrifuged and stored at $-20^{\circ} \mathrm{C}$ until use. Also, the $\mathrm{BI}$ was calculated by counting the acid-fast bacilli in slit-skin smears taken from the earlobe, knee, elbow, and skin lesions and stained by the Ziehl-Neelsen method.

\section{Enzyme-linked immunosorbent assay (ELISA)}

Ninety-six-well polystyrene plates were coated with $2 \mu \mathrm{g} /$ $\mathrm{mL}$ antigen (PGL-1) in sodium carbonate buffer, $\mathrm{pH}$ 9.6, and stored at $4^{\circ} \mathrm{C}$ overnight until use. Serum from each patient was diluted 1:100 in $15 \mathrm{mM}$ Tris-Tween (20 mM Tris, 150 $\mathrm{mM} \mathrm{NaCl}$ and $0.1 \%$ Tween) buffer containing 5\% sheep serum and $10 \mu \mathrm{L}$ was added to each well and the plate was incubated for $1 \mathrm{~h}$ at $37^{\circ} \mathrm{C}$ in a humid chamber. After $1 \mathrm{~h}$, the samples were washed with $15 \mathrm{mM}$ Tris-Tween buffer and anti-human IgM $\beta$-galactosidase conjugate diluted $1: 600$ in $15 \mathrm{mM}$ Tris-Tween buffer containing $5 \%$ sheep serum was added. The plates were then incubated at $37^{\circ} \mathrm{C}$ for $1 \mathrm{~h}$. A fluorigenic substrate $(10 \mu \mathrm{L}$ 4-methylumbelliferyl $\beta$-D-galactopyranoside) was then added to the samples and the material was incubated at $37^{\circ} \mathrm{C}$ for $30 \mathrm{~min}$. The plate was read with a multiskan ELISA reader. Sera with an absorbance at $450 \mathrm{~nm}$ greater than 0.028 (the mean absorbance plus three standard deviations of 35 healthy Brazilian control subjects) were considered to be positive. Each serum was tested in duplicate. The difference between these duplicates was about 3 to $5 \%$. All assays were carried out at the same time (16).

\section{Measurement of the bacterial index}

A reasonably quantitative sample of $M$. leprae from the skin was obtained from slit-skin smears for $\mathrm{BI}$ measurement. The sample was stained by the Ziehl-Neelsen method to determine the density of bacteria in the lesion (BI), based on the count of acid-fast bacilli according to a standard method for the determination of the number of bacilli/field after examining 25-100 fields, on a logarithmic scale ranging from 0-6+ (17).

\section{Statistical analysis}

A mixed linear model (18) was used to compare PGL-1 levels at the two time points (before and after treatment) in each group and the Wilcoxon test (19) was used to compare the $\mathrm{BI}$ at the same time points (before and after treatment).

\section{Results}

A total of 105 leprosy patients were divided into 3 groups: group 1, $34 \mathrm{MB}$ patients treated for 12 months with MDT-MB; group 2, $33 \mathrm{MB}$ patients treated for 24 months with MDT-MB, and group 3, 38 PB patients treated with MDT-PB for 6 months.

Anti-PGL-1 levels were determined before and after 
Table 1. Anti-PGL-1 levels of patients with leprosy before and after treatment.

\begin{tabular}{|c|c|c|c|c|c|c|c|c|c|c|}
\hline Group & Time & Mean \pm SEM & SD & CV & Min & Q1 & Median & Q3 & Max & $\mathrm{P}$ \\
\hline \multirow{2}{*}{ MDT-MB-12 months $(\mathrm{N}=34)$} & Before & $6.95 \pm 1.35$ & 7.85 & 112.97 & 0.20 & 1.10 & 3.20 & 9.60 & 28.0 & $<0.01$ \\
\hline & After & $2.78 \pm 0.69$ & 3.98 & 143.16 & 0.00 & 0.60 & 1.00 & 2.20 & 14.0 & \\
\hline \multirow{2}{*}{ MDT-MB-24 months $(\mathrm{N}=33)$} & Before & $12.53 \pm 2.02$ & 11.60 & 92.61 & 0.60 & 3.90 & 8.80 & 24.20 & 40.0 & $<0.01$ \\
\hline & After & $2.62 \pm 0.79$ & 4.54 & 173.48 & 0.20 & 0.60 & 1.50 & 2.00 & 20.0 & \\
\hline \multirow{2}{*}{ MDT-PB-6 months $(\mathrm{N}=38)$} & Before & $1.28 \pm 0.35$ & 2.17 & 169.92 & 0.00 & 0.40 & 0.70 & 1.40 & 13.5 & \\
\hline & After & $0.62 \pm 0.12$ & 0.74 & 119.53 & 0.00 & 0.30 & 0.50 & 0.70 & 4.5 & \\
\hline
\end{tabular}

Groups: MDT-MB-12 months = multibacillary patients treated for 12 months with multidrug therapy; MDT-MB-24 months = multibacillary patients treated for 24 months with MDT; MDT-PB-6 months $=$ paucibacillary patients treated for 6 months with MDT. CV = coefficient of variation; Min = minimum value; $\mathrm{Q} 1=1$ st quadrant; $\mathrm{Q} 3=3$ rd quadrant; Max = maximum value. $\mathrm{P}<0.01$ comparing values before and after treatment (mixed linear model).

treatment. Untreated MB patients exhibited higher antibody levels (mean \pm SEM): group $1(6.95 \pm 1.35)$ and group 2 $(12.53 \pm 2.02)$ than untreated PB patients $(1.28 \pm 0.35)$. Table 1 shows higher levels of anti-PGL-1 in both groups 1 and 2 before the beginning of the MDT regimens $(6.95 \pm$ 1.35 and $12.53 \pm 2.02$, respectively) and lower levels after the end of MDT regimens $(2.78 \pm 0.69$ and $2.62 \pm 0.79$, respectively). In both groups, the difference between these values was statistically significant $(P<0.01)$, indicating that the short-term MDT regimen led to a reasonable improvement of leprosy patients. In group 3, we found lower levels of anti-PGL-1, which were maintained before and after MDT therapy for 6 months (1.28 \pm 0.35 and $0.62 \pm 0.12$; respectively, Table 1), as expected for PB patients.

The $\mathrm{BI}$ was also sequentially evaluated. PB patients obviously had a negative $\mathrm{BI}$, whereas $\mathrm{MB}$ patients had positive bacillary smears. In groups 1 and 2 , we found higher $\mathrm{BI}$ levels before the beginning of the MDT regimens (1.26 and 1.66, respectively) and lower levels after the end of MDT regimens $(0.26$ and 0.36 , respectively). The differences between these values before and after the MDT regimens were statistically significant in both groups $(P<0.01)$.

Antibody levels were correlated to the $\mathrm{BI}$. The correlation coefficient (Pearson's $r$ ) was 0.72 before and $0.23(P<0.05)$ after treatment in group 1 and 0.67 before and $0.96(P<$ 0.05 ) after treatment in group 2. Since MB patients treated for 12 months with MDT-MB exhibited a large number of negative $\mathrm{BI}(\mathrm{BI}=0)$, we found a lower value of Pearson's $r$ in these patients $(0.23)$.

\section{Discussion}

Since the region of Ribeirão Preto (SP, Brazil) is known to be an area of difficult control of leprosy due to the high migration from different regions, controlled studies to evaluate patients under both short- and long-term chemotherapy are important requirements for the elimination of leprosy as a public health problem.

An important factor predisposing to relapse is inad- equate or irregular therapy, since the duration of the classical treatment regimen ( 24 months) is very long. A shorter treatment regimen (12 months) may improve the adherence of the patients to treatment. Thus, our study compared the outcomes of treatment with 12-month vs 24-month MDT regimens in leprosy patients.

Previous studies comparing the two regimens of leprosy treatment were based on clinical outcomes and $B I$ variations during the course of treatment and after discharge (15). Since serology could provide a method to monitor leprosy patients under treatment (1), in the present study the outcome of leprosy treatment was studied not only on the basis of $\mathrm{BI}$, but also on the basis of anti-PGL-1 levels.

Serology is a marker of a higher systemic bacterial load and may identify potential infectious sources among patients with few clinical signs. It is accepted that there is a correlation between $\mathrm{BI}$ and anti-PGL-1 levels, which could be applied as a serum marker of the patient's bacterial load, presenting high sensitivity and specificity when used for MB leprosy patients $(4,6)$. Our results support this statement, since a significant correlation between anti-PGL-1 levels and bacterial load, measured by the $\mathrm{BI}$, was noted. We observed lower levels of anti-PGL-1 and BI in PB patients independently of treatment. Results from previous PGL-1 studies have demonstrated that the technique is sensitive for MB leprosy but detects only 30 to $60 \%$ of PB cases $(4,6)$. On the other hand, we noted higher levels of antiPGL-1 and $\mathrm{BI}$ in MB patients before the beginning of MDT and a decrease in $\mathrm{BI}$ and anti-PGL-1 levels after the end of treatment, both for the short-term (12 month) and long-term (24 month) regimens.

The seronegative conversion of anti-PGL-1 antibodies is considered useful for monitoring the clearance of the bacterial load, thus measuring the effect of MDT $(4,6,20)$. The present data showed a statistically significant difference in anti-PGL-1 levels determined in MB patients before treatment and after 12 and 24 months of MDT, suggesting that both short-term and long-term MDT regimens led to a reasonable improvement of leprosy patients, in agreement 
with a preliminary study (15). This may reflect clearance of large numbers of bacilli in patients early during treatment (before 12 months), since there is a significant correlation between anti-PGL-1 levels and bacterial load. The tendency of low anti-PGL-1 levels to remain positive may be related to bacillary persistence, a known phenomenon of leprosy. It has been repeatedly shown that macrophages in MB leprosy patients are not able to clear $M$. leprae efficiently from lesions because dead organisms and their fragments continue to persist even after the end of treatment (21). Furthermore, in lepromatous patients under therapy, the duration of treatment seems to be a very important factor for the development of anti-PGL-1 levels, since patients treated for as long as 18 months had positive anti-PGL-1 levels, whereas patients treated for a long time (5 years or more) had normal antibody levels (11). A serologic test for the diagnosis of subclinical infection and for therapeutic follow-up has been applied, despite the high specificity and controversies surrounding the correlation of high levels of anti-PGL-1 antibodies with active, previous or future disease $(11,12)$.

Taken together, the results obtained here using serological testing indicate that the determination of anti-PGL-1 levels not only is a suitable method for the detection of leprosy activity, but is also a rapid, sensitive and inexpensive tool for the evaluation of treatment efficacy $(1,3)$. We

\section{References}

1. Bach MA, Wallach D, Flageul B, Hoffenbach A, Cottenot F. Antibodies to phenolic glycolipid-1 and to whole Mycobacterium leprae in leprosy patients: evolution during therapy. Int J Lepr Other Mycobact Dis 1986; 54: 256-267.

2. Brett SJ, Draper P, Payne SN, Rees RJ. Serological activity of a characteristic phenolic glycolipid from Mycobacterium leprae in sera from patients with leprosy and tuberculosis. Clin Exp Immunol 1983; 52: 271-279.

3. Foss NT, Callera F, Alberto FL. Anti-PGL1 levels in leprosy patients and their contacts. Braz J Med Biol Res 1993; 26: 43-51.

4. Klatsewr PR. Serology of leprosy. Trop Geogr Med 1994; 46: 115-118.

5. Saad MH, Medeiros MA, Gallo ME, Fonseca LS. Use of the anti-PGL-I antibody ELISA and the Mitsuda reaction in early diagnosis of leprosy. Braz J Med Biol Res 1991; 24: 801-805.

6. Stefani MM, Martelli CM, Morais-Neto OL, Martelli P, Costa MB, de Andrade AL. Assessment of anti-PGL-I as a prognostic marker of leprosy reaction. Int $J$ Lepr Other Mycobact Dis 1998; 66: 356-364.

7. Bakker MI, Hatta M, Kwenang A, Van Mosseveld P, Faber WR, Klatser PR, et al. Risk factors for developing leprosy - a population-based cohort study in Indonesia. Lepr Rev 2006; 77: 48-61.

8. Brasil MTLRF, Oliveira LR, Rímoli NS, Cavallari FS, Gonçalves OS, Lessa ZL, et al. Anti-PGL-1 serology and the risk of leprosy in a highly endemic area in the State of São Paulo, conclude that sequential serum measurements are helpful to understand the dynamics of antibody production during the course of leprosy. Our study permitted us to determine a clear antibody profile directly associated with the BI.

Follow-up studies of MB patients treated for 12 and 24 months with MDT indicated that the majority of patients tested negative for $M$. leprae both in skin washings and nasal secretions as determined by direct microscopic observation and PCR. The same patients remained $\mathrm{BI}$ positive when analyzed by routine slit-skin smears and biopsy after 1 year of MDT (21). Since the present study showed seronegative conversion of anti-PGL-1 antibodies in MB patients treated with MDT for both 12 and 24 months, it is possible that the control of leprosy infection can be obtained with 12 months of MDT in MB patients, relieving patients of long treatment periods. Thus, the adherence of the patients to the treatment should improve, leading to the elimination of viable $M$. leprae rapidly and blocking the transmission of the infection. Based on these observations, a reduction in length of chemotherapy may facilitate patient compliance and national health program activities.

\section{Acknowledgments}

The authors thank Mr. Mario Ignacio Neto for technical assistance.

Brazil: four-year follow-up. Rev Bras Epidemiol 2003; 6: 262-271.

9. Cho SN, Yanagihara DL, Hunter SW, Gelber RH, Brennan PJ. Serological specificity of phenolic glycolipid I from Mycobacterium leprae and use in serodiagnosis of leprosy. Infect Immun 1983; 41: 1077-1083.

10. Burgess PJ, Fine PE, Ponnighaus JM, Draper C. Serological tests in leprosy. The sensitivity, specificity and predictive value of ELISA tests based on phenolic glycolipid antigens, and the implications for their use in epidemiological studies. Epidemiol Infect 1988; 101: 159-171.

11. Douglas JT, Steven LM, Fajardo T, Cellona RV, Madarang MG, Abalos RM, et al. The effects of chemotherapy on antibody levels in lepromatous patients. Lepr Rev 1988; 59: 127-135.

12. Barros RPC, Oliveira MLWR. Detection of specific antibodies for the phenolic-1 glycolipid antigen of $M$. leprae (anti-PGL-1 IgM): applications and limitations. An Bras Dermatol 2000; 75: 745-753.

13. Ministério da Saúde - Brasil. Secretaria de Vigilância em Saúde. Programa Nacional de Eliminação da Hanseníase. Situação da Hanseníase no Brasil. Prevalência e Detecção no ano de 2007. Brasília: Ministério da Saúde, PNEH/ DEVEP/SVS/MS; 2008.

14. Ministério da Saúde - Brasil. Portaria No 817 de 26 de julho de 2000 DO 144-E, de 27 de julho de 2000. Brasília: Ministério da Saúde; 2000.

15. Sales AM, Sabroza PC, Nery JA, Duppre NC, Sarno EN. No 
difference in leprosy treatment outcomes comparing 12- and 24-dose multidrug regimens: a preliminary study. Cad Saude Publica 2007; 23: 815-822.

16. Bonato VLD. Correlação dos anticorpos anti-PGL-I com o índice baciloscópico, a reação de Mitsuda, o tratamento poliquimioterápico e as interleucinas nas diferentes formas da hanseníase. [Master's thesis]: Universidade de São Paulo; 1995.

17. Ridley DS. Therapeutic trials in leprosy using serial biopsies. Lepr Rev 1958; 29: 45-52.

18. McLean RA, Sanders WL, Stroup WW. A unified approach to mixed linear models. Am Stat 1991; 45: 54-64.
19. Conover WJ. Practical nonparametric statistics. 3rd edn. New York: Wiley; 1999.

20. Meeker HC, Schuller-Levis G, Fusco F, Giardina-Becket MA, Sersen E, Levis WR. Sequential monitoring of leprosy patients with serum antibody levels to phenolic glycolipid-I, a synthetic analog of phenolic glycolipid-I, and mycobacterial lipoarabinomannan. Int J Lepr Other Mycobact Dis 1990; 58: 503-511.

21. Job CK, Jayakumar J, Kearney M, Gillis TP. Transmission of leprosy: a study of skin and nasal secretions of household contacts of leprosy patients using PCR. Am J Trop Med Hyg 2008; 78: 518-521. 